\title{
Algoritmo de Luus-Jaakola Aplicado a um Problema Inverso de Fermentação Batelada Alimentada ${ }^{1}$
}

F.S. LOBATO ${ }^{2}$, V. STEFFEN JR${ }^{3}$, Faculdade de Engenharia Mecânica, Universidade Federal de Uberlândia, Campus Santa Mônica, 38400-902 Uberlândia, MG, Brasil.

Resumo. O presente trabalho apresenta um estudo sobre a estimação de parâmetros cinéticos em processo de fermentação batelada alimentada usando o Método de Luus-Jaakola modificado. Nesta versão do algoritmo, são realizadas reduções individuais no espaço de busca para cada variável de projeto. Neste sentido, apresentase uma breve revisão sobre a técnica de otimização e, em seguida, o algoritmo é testado em um problema de fermentação batelada alimentada. Os resultados obtidos são comparados com a técnica de Evolução Diferencial e mostraram que a metodologia se configura como uma alternativa promissora para a proposta mencionada acima.

Palavras-chave. Algoritmo de Luus-Jaakola, Fermentação Alcoólica, Problema Inverso.

\section{Introdução}

O Problema de Identificação de Parâmetros ou Problema Inverso (PI), surge da necessidade de obtenção de parâmetros de modelos teóricos que são ajustados pela comparação com dados experimentais confiáveis, de forma que possam ser utilizados para simular o comportamento do sistema para diferentes condições de operação. Evita-se assim, a necessidade da realização de experimentos específicos para uma dada condição [11]. Tradicionalmente, este tipo de problema tem sido tratado por técnicas de otimização determinísticas $[2,4]$. Entretanto, nos últimos anos, o uso de técnicas não determinísticas ou o acoplamento destas com outras técnicas vem ganhando importância devido à sua "simplicidade" conceitual $[5,11,18,21]$.

Dentre os inúmeros métodos estocásticos de busca, o algoritmo de Luus-Jaakola (LJ) [14], proposto inicialmente para a resolução de prolemas de engenharia química $[13,14,17]$, se configura como um dos métodos de busca aleatória mais populares que existem. Atualmente pode-se enumerar aplicações do algoritmo de LJ em diversas áreas, tais como, problemas de controle ótimo com arcos singulares [12, 13],

\footnotetext{
${ }^{1}$ Os autores agradecem ao CNPq (Proc 470346/2006-0) pelo financiamento parcial desta pesquisa.

${ }^{2}$ franpi22@yahoo.com.br

${ }^{3}$ vsteffen@mecanica.ufu.br
} 
cromatografia [16], estimação de propriedades radiativas [9], resolução de funções matemáticas multimodais [6], dentre outras aplicações.

Nos últimos anos, esforços têm sido direcionados no sentido de aumentar o desempenho do algoritmo de LJ através do desenvolvimento de metodologias mais eficientes para a redução do espaço de busca. Luus e Hennessy [13] propuseram o procedimento de multi-passos, no qual a região de busca para cada variável de projeto é atualizada segundo o valor calculado entre a passagem do laço interno para o externo. Mais recentemente, foi desenvolvida uma abordagem mais sofisticada para a redução do espaço de busca, através da atualização dos coeficientes de contração em cada looping do algoritmo [8].

Uma das áreas de maior relevância na engenharia química é a estimação de parâmetros cinéticos em processos biotecnológicos considerando a operação batelada e a batelada alimentada. Nesses processos faz-se necessária a modelagem matemática de equações cinéticas de crescimento de substrato e de micro-organismos (células) e da produtividade para o produto, considerando-se a natureza inibitória oriunda das altas concentrações dos participantes [1, 3, 10, 21].

Este trabalho está estruturado conforme segue. A seção 2 descreve o processo de fermentação batelada alimentada. A seção 3 traz uma breve revisão sobre o algoritmo de LJ. Já a seção 4 mostra a formulação matemática do PI. Na seção 5, é apresentada uma análise da influência de parâmetros do algoritmo de LJ na qualidade da solução, comparando-a com o algoritmo de Evolução Diferencial (ED) [19]. As conclusões e sugestões para trabalhos futuros são apresentadas na seção 6 .

\section{O Processo de Fermentação Batelada Alimen- tada}

Do ponto de vista prático, a determinação do modo de alimentação de substrato no processo de fermentação batelada alimentada se configura como um dos maiores desafios em biotecnologia devido à dependência dos modelos cinéticos, das condições iniciais e da sua natureza singular $[1,3,7,10,12,13,15,21]$.

O processo de fermentação batalada alimentada é formado pelo comportamento dinâmico das equações de balanço de massa

$$
\begin{gathered}
\dot{x}=\mu x-\frac{u}{v} x \quad x(0)=x_{o} \\
\dot{s}=-\sigma x+\frac{u}{v}\left(s_{f}-s\right) \quad s(0)=s_{o} \\
\dot{p}=\pi x-\frac{u}{v} p \quad p(0)=p_{o} \\
\dot{v}=u \quad v(0)=v_{o}
\end{gathered}
$$

onde $x, s$ e $p$ são as concentrações de células, substrato e produto, respectivamente, $u$ é a taxa de alimentação de substrato, $s_{f}$ é a concentração da corrente de substrato alimentada e $v$ é o volume do fermentador. As características do micro-organismo utilizado no processo de fermentação, da forma de crescimento ou decrescimento 
de substrato, célula e de produto e seus efeitos inibitórios são representados pelas taxas de crescimento específica $\mu, \sigma$ e $\pi$.

Neste trabalho serão consideradas as seguintes expressões para $\mu, \sigma$ e $\pi$, válidas para $0 \mathrm{~g} / \mathrm{L} \leq s \leq 200 \mathrm{~g} / \mathrm{L}$ e $0 \mathrm{~g} / \mathrm{L} \leq p \leq 100 \mathrm{~g} / \mathrm{L}$ [20]:

$$
\begin{gathered}
\mu=\mu_{\max }\left(\frac{s}{K_{s}+s+\frac{s^{2}}{K_{i}}}\right)\left(1-\frac{p}{p_{\max }}\right)^{n} \\
\pi=\frac{1}{y_{x s}} \mu \\
\sigma=\frac{y_{p s}}{y_{x s}} \mu,
\end{gathered}
$$

onde $\mu_{\max }$ é a taxa específica máxima de crescimento celular, $K_{s}$ é a constante de saturação para o crescimento celular, $K_{i}$ é a constante de inibição do crescimento celular pelo substrato, $p_{\max }$ é a concentração de produto onde cessa o crescimento microbiano, $m s$ é a constante de manutenção celular, $n$ é a potência do termo de inibição pelo produto, $y_{x s}$ e $y_{p s}$ são os rendimentos de células/substrato e produto/substrato, respecivamente.

Fisicamente, também são impostas restrições à variável de controle e para o volume

$$
\begin{gathered}
0=u_{\min } \leq u \leq u_{\max } \\
v \leq v_{\max } .
\end{gathered}
$$

O modelo descrito acima é adimimensionalizado através do uso dos seguintes grupos [3]:

$x=\frac{X}{X_{\max }} \frac{[g \text { célula }]}{[g \text { célula }]} ; s=\frac{S}{S_{f}} \frac{[g \text { substrato }]}{[g \text { substrato }]} ; p=\frac{P}{P_{\max }} \frac{[g \text { produto }]}{[g \text { produto }]} ; v=\frac{V}{V_{\max }} \frac{[L]}{[L]} ;$

$t=\frac{\tau}{\tau_{\max }} \frac{[h]}{[h]} ; u=U \frac{\tau_{\max }}{V_{\max }} \frac{[L]}{[h]} \frac{[h]}{[L]} ; y_{x s}=Y_{x s} \frac{S_{f}}{X_{\max }} \frac{[-]}{[-]} ; y_{p s}=Y_{p s} \frac{S_{f}}{P_{\max }} \frac{[-]}{[-]} ;$

$s_{f}=\frac{S_{f}}{S_{f}} \frac{[-]}{[-]} ; \mu_{\max }=\mu_{m} \tau_{\max } \frac{[h]}{[h]} ; m s=M_{s} \tau_{\max } \frac{[h]}{[h]} ; K_{s}=\frac{k_{s}}{S_{f}} \frac{\text { [conc. células }]}{[\text { conc. células }]} ;$

$K_{i}=\frac{k_{i}}{S_{f}} \frac{[\text { conc. células }]}{[\text { conc. células }]} ; p_{\max }=\frac{P_{m}}{P_{\max }} \frac{[g \text { produto }]}{[g \text { produto }]}$.

As grandezas $X_{\max }, S_{f}, V_{\max }, P_{\max }, \tau_{\max }, \mu_{m}, k_{i}, k_{s}$ e $M_{s}$ são os valores reais das concentrações, do volume, do controle e dos parâmetros para cada experimento realizado, descritos em detalhes no trabalho de Borges [3].

\section{O Algoritmo de Luus-Jaakola}

O algoritmo de LJ foi proposto em 1973 para a solução de problemas de otimização pelos autores que emprestaram seus nomes à técnica, Luus e Jaakola [14]. Na primeira versão do algoritmo, a atualização do projeto ótimo era realizada ao contrairse a região de busca. Posteriormente, o próprio autor passaria a atualizar o projeto ótimo imediatamente após uma solução melhor ter sido achada, como descrito a seguir [13]: 
- PAsso 1: escolher um projeto inicial $x^{*}$ (aleatória ou não) com soluções factíveis para o problema em questão, onde garante-se por "regras de reparo" que os valores atribuídos às variáveis estão dentro das fronteiras delimitadas pelo projetista; escolher uma região de busca inicial de tamanho $r$, o número de laços internos $\left(n_{\text {int }}\right)$, o número de laços externos $\left(n_{\text {ext }}\right)$ e o coeficiente de contração $\epsilon$;

- fazer $i=1\left(i=1, \ldots, n_{\text {ext }}\right)$ (Início do laço externo) e $j=1\left(j=1, \ldots, n_{\text {int }}\right)$ (Início do laço interno);

- PAsso 2: enquanto $j<=n_{\text {int }}$ atualizar o projeto atual através da relação $x=x^{*}+D r$, onde $D$ é uma matriz diagonal com elementos aleatórios entre $-0,5$ e 0,5 . Verificar a viabilidade desse projeto através das "regras de reparo". Se o projeto resultante $x$ apresenta uma função de aptidão melhor que $x^{*}$, atualizar $x^{*}\left(x^{*}=x\right)$; caso contrário, o projeto não é atualizado (Fim do laço interno);

- contrair a região de busca $r(r=(1-\epsilon) r)$, atualizar o valor de $i(i=i+1)$, fazer $j=1$ e prosseguir para o PAsso 2 ;

- Fim do laço externo $\rightarrow$ projeto ótimo $x^{*}$.

Visando aumentar o desempenho desse algoritmo, Luus [12] propôs a atualização da região de busca através dos desvios apresentados por cada variável de projeto, da seguinte forma: após completado o laço interno $j$, isto é $j=n_{\text {int }}$, modifica-se a região de busca $r$ segundo:

$$
r=\left|x_{n_{\text {int }}}^{*}-x_{j}^{*}\right|,
$$

onde $x_{n_{\text {int }}}^{*}$ é o melhor projeto ao final de um laço interno completo e $x_{j}^{*}$ é o melhor projeto ao final de um laço externo completo. Com esse mecanismo, consegue-se atualizar a região de busca para cada variável de projeto. Se a região de uma determinada variável é nula, deve-se assumir que a região é representada por um valor bem pequeno, nesse caso usa-se $10^{-6}$ para a variável em questão.

\section{Formulação Matemática do Problema Inverso}

O problema inverso é definido como sendo o somatório dos desvios entre os valores experimentais e os valores calculados pelo sistema de equações (2.1) a (2.9)

$$
f=\sum_{j=1}^{M} \sum_{i=1}^{N_{j}}\left(\left(x_{e x p}^{j, i}-x_{c a l}^{j, i}\right)^{2}+\left(s_{e x p}^{j, i}-s_{c a l}^{j, i}\right)^{2}+\left(p_{e x p}^{j, i}-p_{c a l}^{j, i}\right)^{2}\right),
$$

onde $\theta_{c a l}^{j, i}$ e $\theta_{\text {exp }}^{j, i}$ são os valores calculados e experimentais para $\theta\left(\theta=\left[\begin{array}{lll}x & s & p\end{array}\right]\right)$ no experimento $j(j=1, \ldots, M)$ e $N_{j}$ é o número de pontos do experimento $j$. Desse modo, nosso objetivo é a determinação dos valores de $\mu_{\max }, K_{s}, K_{i}, p_{\max }, n$ e $m s$ que minimizem $f$ e que não violem as seguintes desigualdades [3]: $0,001 \leq \mu_{\max } \leq 10$; $3 \times 10^{-5} \leq K_{s} \leq 0,12 ; 6 \times 10^{-4} \leq K_{i} \leq 4,0 ; 0,7 \leq p_{\max } \leq 1,90 ; 0,10 \leq n \leq 4,0 \mathrm{e}$ $0 \leq m s \leq 8,0$. 


\section{Resultados e Discussões}

Com o intuito de se avaliar a potencialidade do algoritmo de LJ modificado na resolução do problema inverso, serão utilizados os pontos experimentais referentes às concentrações de substrato na corrente de alimentação $\left(S_{f}\right)$ de 216,66 g/L, 241,42 $\mathrm{g} / \mathrm{L}$ e 285,11 g/L obtidos por Borges [3] e mostrados na Tabela 1.

Tabela 1: Condições iniciais e de operação para cada um dos experimentos.

\begin{tabular}{|c|c|c|c|c|c|c|c|c|c|}
\hline Exp. & $x_{o}$ & $s_{o}$ & $p_{o}$ & $v_{o}$ & $y_{x s}$ & $y_{p s}$ & $t_{e}$ & $s_{f}$ & $u_{\max }$ \\
\hline \hline 1 & 0 & 1 & 0,4534 & 0,3008 & 0,0594 & 1,2425 & 0,6604 & 1 & 1,0586 \\
\hline 2 & 0 & 1 & 0,4520 & 0,3007 & 0,0623 & 1,2423 & 0,5216 & 1 & 1,3403 \\
\hline 3 & 0 & 1 & 0,4019 & 0,3009 & 0,0584 & 1,2869 & 0,4727 & 1 & 1,4788 \\
\hline
\end{tabular}

Deve ser destacado que na metodologia experimental utilizada em [3], o processo experimental inicializa-se com $u=u_{\max }$ e prossegue até que o reator alcance o seu volume máximo ( $v=v_{\max }=1$, no modelo adimensionalizado). A partir desse instante $t_{e}$ (tempo de enchimento), a vazão de alimentação de substrato é cortada, isto é, $u=u_{\min }=0$. Assim, ficam claramente definidas duas regiões, uma batelada alimentada e outra batelada.

A Tabela 2 apresenta os resultados para 10 execuções do algortimo de LJ modificado para a obtenção do melhor projeto $\left(\varphi=\left[\mu_{\max } K_{s} K_{i} p_{\max } n m s\right]\right)$, dos valores médios $(\bar{\varphi})$ e do desvio padrão $(\Delta)$. Esses resultados são comparados com os obtidos por Borges [3] usando ED com 20 indivíduos na população, taxa de perturbação e probabilidade de cruzamento ambos iguais a 0,8 e 250 gerações, totalizando assim 5020 avaliações da função objetivo. Para comparar os dois algoritmos foi escolhida uma combinação do número de laços internos $\left(n_{\text {int }}\right)$ e externos $\left(n_{\text {ext }}\right)$ de tal forma que o número de avaliações do algoritmo de LJ não ultrapasse o número de avaliações do algoritmo de ED utilizado por Borges [3]. 
Tabela 2: Resultados obtidos pelo Algoritmo de Luus-Jaakola (LJ) e pelo Algoritmo de Evolução Diferencial (ED). NFO é o número de avaliações da função objetivo.

\begin{tabular}{|c|c|c|c|c|c|c|c|c|c|c|c|c|}
\hline Caso & $n_{\text {ext }}$ & $n_{\text {int }}$ & $\epsilon$ & & $\mu_{\max }$ & $K_{s}$ & $K_{i}$ & $p_{\max }$ & $n$ & $m s$ & $f$ & NFO \\
\hline \multirow{3}{*}{1} & \multirow{3}{*}{20} & \multirow{3}{*}{250} & \multirow{3}{*}{0,01} & $\varphi$ & 0,292171 & 0,095368 & 0,809521 & 1,798327 & 0,115534 & 0,045900 & 0,033015 & \multirow{3}{*}{5001} \\
\hline & & & & $\bar{\varphi}$ & 0,304775 & 0,101277 & 0,725346 & 1,674618 & 0,111527 & 0,045229 & 0,033077 & \\
\hline & & & & & 0,017904 & 0,009437 & 0,121869 & 0,202929 & 0,006764 & 0,009220 & 0,000064 & \\
\hline \multirow{3}{*}{2} & \multirow{3}{*}{20} & \multirow{3}{*}{250} & \multirow{3}{*}{0,05} & $\varphi$ & 0,296518 & 0,092890 & 0,785774 & 1,630906 & 0,140661 & 0,051072 & 0,033230 & \multirow{3}{*}{5001} \\
\hline & & & & $\bar{\varphi}$ & 0,304240 & 0,099021 & 0,741054 & 1,570747 & 0,122464 & 0,045237 & 0,033160 & \\
\hline & & & & $\Delta$ & 0,013176 & 0,007373 & 0,067881 & 0,211704 & 0,011663 & 0,006619 & 0,000159 & \\
\hline \multirow{3}{*}{3} & \multirow{3}{*}{50} & \multirow{3}{*}{100} & \multirow{3}{*}{0,01} & $\varphi$ & 0,328815 & 0,113565 & 0,574566 & 1,696371 & 0,127136 & 0,044083 & 0,032978 & \multirow{3}{*}{5001} \\
\hline & & & & $\bar{\varphi}$ & 0,318715 & 0,107730 & 0,640107 & 1,739375 & 0,135873 & 0,045037 & 0,033098 & \\
\hline & & & & $\Delta$ & 0,011994 & 0,007140 & 0,054734 & 0,179316 & 0,007757 & 0,009019 & 0,000126 & \\
\hline \multirow{3}{*}{4} & \multirow{3}{*}{50} & \multirow{3}{*}{100} & \multirow{3}{*}{0,05} & $\varphi$ & 0,325984 & 0,117168 & 0,593596 & 1,734553 & 0,100056 & 0,037927 & 0,032898 & \multirow{3}{*}{5001} \\
\hline & & & & $\bar{\varphi}$ & 0,316187 & 0,105919 & 0,670597 & 1,552461 & 0,123417 & 0,041102 & 0,033111 & \\
\hline & & & & $\Delta$ & 0,01 & 0,010125 & 0,103798 & 0,14 & 0,017186 & 0,006690 & 0,000147 & \\
\hline \multirow{3}{*}{5} & \multirow{3}{*}{100} & \multirow{3}{*}{50} & \multirow{3}{*}{0,01} & $\varphi$ & 0,327100 & 0,112774 & 0,612392 & 1,692249 & 0,146303 & 0,041163 & 0,033056 & \multirow{3}{*}{5001} \\
\hline & & & & $\bar{\varphi}$ & 0,315625 & 0,104096 & 0,692260 & 1,601673 & 0,138919 & 0,043848 & 0,033183 & \\
\hline & & & & $\Delta$ & 0,028281 & 0,014998 & 0,148013 & 0,187712 & 0,009292 & 0,007973 & 0,000091 & \\
\hline \multirow{3}{*}{6} & \multirow{3}{*}{100} & \multirow{3}{*}{50} & \multirow{3}{*}{0,05} & $\varphi$ & 0,308657 & 0,106553 & 0,705935 & 1,765776 & 0,114232 & 0,037426 & 0,033000 & \multirow{3}{*}{5001} \\
\hline & & & & $\bar{\varphi}$ & 0,311186 & 0,102800 & 0,692718 & 1,493287 & 0,117622 & 0,042431 & 0,033122 & \\
\hline & & & & $\Delta$ & 0,009127 & 0,005542 & 0,055383 & 0,251798 & 0,017549 & 0,005365 & 0,000091 & \\
\hline & & & & $\varphi$ & 0,307395 & 0,104409 & 0,693030 & 1,775701 & 0,111726 & 0,038710 & 0,032980 & \\
\hline 7 & 200 & 25 & 0,01 & $\bar{\varphi}$ & 0,303960 & 0,098505 & 0,737048 & 1,573094 & 0,121817 & 0,046680 & 0,033190 & 5001 \\
\hline & & & & $\Delta$ & 0,012643 & 0,006621 & 0,074506 & 0,215048 & 0,011226 & 0,008584 & 0,000182 & \\
\hline & & & & $\varphi$ & 0,329427 & 0,115620 & 0,586129 & 1,713606 & 0,128174 & 0,044656 & 0,032960 & \\
\hline 8 & 200 & 25 & 0,05 & $\bar{\varphi}$ & 0,319260 & 0,107405 & 0,640026 & 1,668971 & 0,134778 & 0,045189 & 0,033144 & 5001 \\
\hline & & & & $\Delta$ & 0,011833 & 0,007782 & 0,048381 & 0,195849 & 0,009282 & 0,010061 & 0,000181 & \\
\hline ED [3] & - & - & - & - & 0,30852 & 0,10588 & 0,69178 & 1,61198 & 0,10000 & 0,04257 & 0,032979 & 5020 \\
\hline
\end{tabular}


Na Tabela 2 quando se compara o valor da função objetivo obtido pelo algoritmo de LJ com o algoritmo de ED percebe-se que estes valores estão na mesma ordem de grandeza, isto é, são bastante coerentes. Todavia, deve-se destacar que no algoritmo de LJ, são necessárias 5001 avaliações da função objetivo contra 5020 avaliações da função objetivo usando o algoritmo de ED. Com relação à variação do número de laços internos e externos do algoritmo pode-se afirmar que, para o problema em questão, estes pouco influenciaram o valor da função objetivo. Neste sentido, uma conclusão definitiva sobre a influência destes parâmetros não pode ser feita.

As Figuras 1 e 2 apresentam os perfis de concentração de células, de substrato e de produto e volume do reator para o menor valor de função objetivo obtido pelo algoritmo de LJ, isto é, o caso 4.

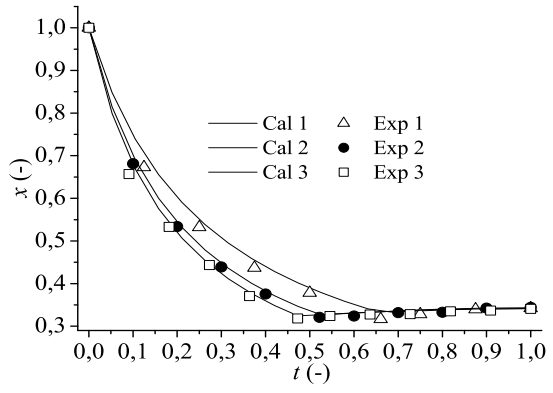

(a) $x(-)$

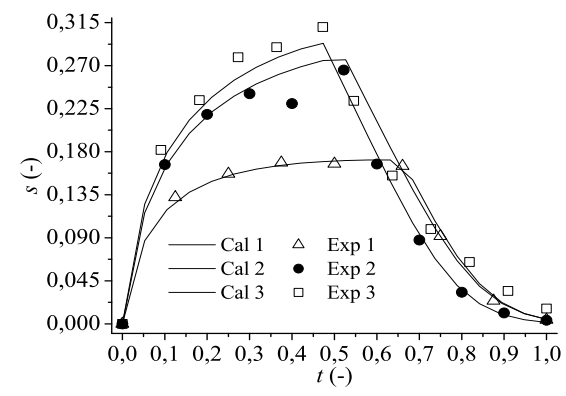

(b) $s(-)$

Figura 1: Perfis de concentração de células e de substrato.

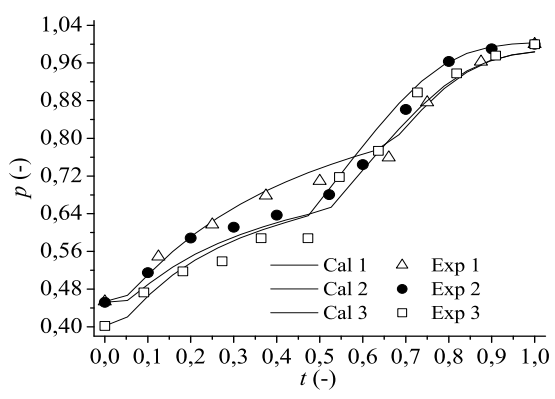

(a) $p(-)$

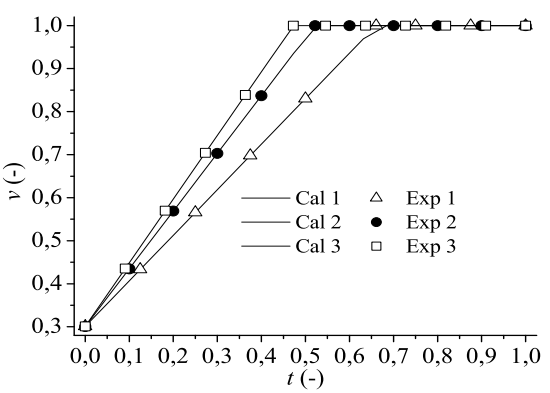

(b) $v(-)$

Figura 2: Perfis de concentração de produto e volume do fermentador.

A partir da análise dessas figuras pode-se perceber que a maior contribuição para o aumento no valor da função objetivo $f$ se deve às concentrações experimentais de substrato e de produto. É claro que, como se trata de pontos experimentais, deve-se levar em conta a qualidade de obtenção dos mesmos, já que erros de medida e outros 
aspectos são inerentes a tais procedimentos. Além disso, deve ser ressaltado que o volume não foi considerado na elaboração de $f$, pois, como o volume teoricamente só depende de $u$ e esse é conhecido e constante ao longo do processo ( $u$ é $u_{\max }$ ou $u$ é $u_{\min }=0$ ), implica que a equação 2.4 sempre terá essa qualidade de solução, isto é, a sua contribuição na função objetivo sempre será muito próxima de zero.

Uma observação muito oportuna a ser feita é o alto desvio padrão de $p_{\max }$, quando se compara com os desvios de todas as outras variáveis de projeto. Esse alto desvio nos induz a pensar em uma alta sensibilidade do modelo com relação a esse parâmetro. Tal sensibilidade pode ser observada na Figura 3 quando se varia apenas $p_{\max }$ para $60 \%$ abaixo e acima do melhor valor obtido, isto é, o caso 4 (ver Tabela 2), mantendo fixos os valores dos demais.

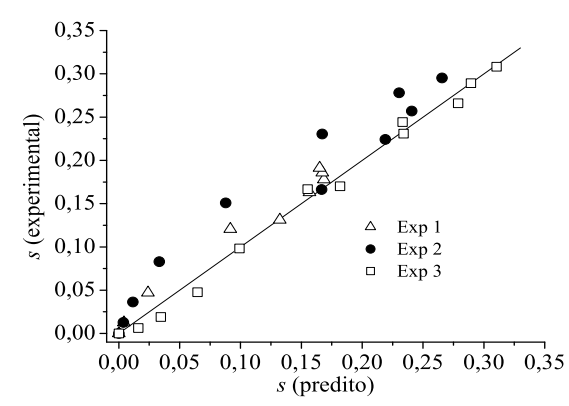

(a) 0,60 de $p_{\max }$

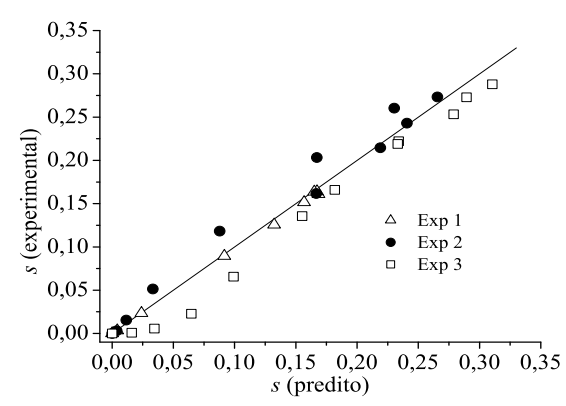

(b) 1,60 de $p_{\max }$

Figura 3: Perfis de concentração de substrato com variação de $p_{\max }$.

\section{Conclusões e Sugestões}

Este trabalho objetivou a estimação de propriedades cinéticas através do uso do algoritmo de LJ modificado. Esta versão leva em consideração regiões de busca diferentes para cada uma das variáveis de projeto.

De forma geral, os resultados obtidos mostram que a técnica empregada se configura como uma alternativa promissora para a finalidade a que esse trabalho se propôe. Deve-se ressaltar que, em comparação com técnicas heurísticas como os Algoritmos Genéticos e a Evolução Diferencial, o algoritmo de LJ não apresenta um aumento no número de parâmetros de controle, o que configura uma vantagem. Além disso, a variação do número de laços internos e externos do algoritmo de LJ, para o problema estudado, mostrou que pode-se escolher algumas combinações destes parâmetros sem perda de qualidade da solução, quando comparado ao algoritmo de ED.

Como linha de trabalhos futuros, pode-se citar a análise da sensibilidade dos parâmetros do modelo através do acoplamento e resolução das equações de sensibilidade. Pode-se considerar também a hibridização do algoritmo de LJ com outros 
métodos de otimização (determinísticos ou não) e a exploração de outros mecanismos para a atualização da região de busca.

\begin{abstract}
This work presents a study regarding the estimation of kinetics parameters in a fed-batch fermentation process using a Modified Luus-Jaakola Method. In this version of the algorithm, individual reductions are performed for each design variable. In this way, a review about the Luus-Jaakola optimization technique is presented. Then, the algorithm is tested in a feed-batch fermentation problem. The obtained results are compared with those obtained from the Differential Evolution technique. This comparison demonstrated that the methodology used represents a promising alternative for the problem studied.
\end{abstract}

Key-words. Luus-Jaakola Algorithm, Alcohol Fermentation, Inverse Problem.

\title{
Referências
}

[1] S. Aiba, M. Shoda, M. Nagatani, Kinetics of product inhibition in alcohol fermentation, Biotechnology and Bioengineering, X (1968), 845-864.

[2] M. Baltes, R. Schneider, C. Sturm, M. Reuss, Optimal experimental design for parameter estimation in unstructured growth models, Biotechnology Programming, 10 (1994), 480-491.

[3] P.C.S. Borges, "Otimização Dinâmica da Fermentação Alcoólica no Processo em Batelada Alimentada", Dissertação de Mestrado, Faculdade de Engenharia Química, Universidade Federal de Uberlândia, 2008.

[4] L. Cazzador, V. Lubenova, Nonlinear estimation of specific growth rate for aerobic fermentation processes, Biotechnology Programming, 47 (1995), 626634 .

[5] E.S. Chalhoub, H.F., Campos Velho, A.J. Silva-Neto, A comparison of the onedimensional radiative transfer problem solutions obtained with the Monte Carlo method and three variations of the discrete ordinates method, "Proceedings of $19^{\text {th }}$ International Congress of Mechanical Engineering", ABCM, Brasília, Brazil, 2007.

[6] A.C.R. Coelho, H. Henderson, W.F. Sacco, R.P. Domingos, Comparasion of Luus-Jaakola algorithm and particle swarm optimization applied to a multimodal test function, "X Encontro de Modelagem Computacional", Instituto Politécnico - UERJ, Nova Friburgo, Rio de Janeiro, 2007.

[7] J. Hong, Optimal substrate feeding policy for a fed batch fermentation with substrate and product inhibition kinetics, Biotechnology and Bioengineering, XXVIII, (1986), 1421-1431.

[8] J. Jezowski, R. Bochenek, G. Ziomek, Random search optimization approach for highly multi-modal nonlinear problems, Advances in Engineering Software, 36, (2005), 504-517. 
[9] D.C. Knupp, A.J. Silva-Neto, W.F. Sacco, Estimation of radiative properties with the Luus-Jaakola method, "X Encontro de Modelagem Computacional", Instituto Politécnico - UERJ, Nova Friburgo, Rio de Janeiro, 2007.

[10] F.S. Lobato, L.C. Oliveira-Lopes, V.V. Murata, Optimal feed policy for fedbath fermentation with events identification based on switching structures, "XXII IACChE (CIIQ)2006/VCAIQ", 2006.

[11] F.S. Lobato, A.J. Silva-Neto, V. Steffen Jr, Um estudo comparativo usando evolução diferencial e recozimento simulado aplicado a um problema inverso de transferência radiativa, "X Encontro de Modelagem Computacional", Instituto Politécnico - UERJ, Nova Friburgo, Rio de Janeiro, 2007.

[12] R. Luus, Use of Luus-Jaakola optimization procedure for singular optimal control problems, Nonlinear Analysis, 47, (2001), 5647-5658.

[13] R. Luus, D. Hennessy, Optimization of fed-batch reactors by the Luus-Jaakola optimization procedure, Industrial Engineering Chemical Research, 38, (1999), 1948-1955.

[14] R. Luus, T.H.I. Jaakola, Optimization by direct search and systematic reduction of the size of search region, AIChE Journal, 19, (1973), 760-766.

[15] J.M. Modak, H.C. Lim, Y.J. Tayeb, General characteristics of optimal feed rate profiles for various fed-batch fermentation processes, Biotechnology and Bioengineering, XXVIII, (1986), 1396-1407.

[16] I. Poplewska, W. Piatkowski, D. Antos, Effect of temperature on competitive adsorption of the solute and the organic solvent in reversed-phase liquid chromatography, Journal of Chromatography, 1103 (2006), 284-295.

[17] R.L. Salcedo, M.J. Gonçalves, S. F. Azevedo, An improved random search algorithm for non-linear optimization, Computers and Chemical Engineering, 14 (1990), 1111-1126.

[18] A.J. Silva-Neto, F.J.C.P. Soeiro, Estimation of the phase function of anisotropic scattering with a combination of gradient based and stochastic global optimization methods, "Proceedings of $5^{\text {th }}$ World Congress on Computational Mechanics", Vienna, Austria, July, 7-12, 2002.

[19] R. Storn, K. Price, Differential evolution: a simple and efficient adaptive scheme for global optimization over continuous spaces, International Computer Science Institute, 12 (1995), 1-16.

[20] G.M. Tosetto, "Influência da Matéria-Prima no Comportamento Cinético de Levedura na Produção de Etanol", Dissertação de Mestrado, Faculdade de Engenharia Química, Universidade Estadual de Campinas, 2002.

[21] F.S. Wang, T.L. Su, H.J. Jang, Hybrid differential evolution for problems of kinetic parameter estimation and dynamic optimization of an ethanol fermentation process, Industry Engineering Chemical Research, 40 (2001), 2876-2885. 